181. Webster, Jr., W. J., Wink, J. E., Altenhoff, W. J. 1970, Astrophys. Letters, 7, 47.

182. Webster, W. J., Altenhoff, W. J., Wink, J. E. $\quad 1971$, Astron. J., 76, 677.

183. Weiler, K. W., Seielstad, G. A. $\quad 1971$, Astrophys. J., 163, 455.

184. Weiler, K. W., Seielstad, G. A. $\quad 1972$, Astron. Astrophys, $21,393$.

185. Wendker, H. J. 1970, Astron. Astrophys., 4, 378.

186. Wendker, H. J., Dickel, J. R., Yang, K. S., and Staff (Vermilion River Observatory, University of Illinois) 1970, Astron. J., 75, 148.

187. Wendker, H. J. 1971, Astron. Astrophys., 13, 65.

188. Willis, A. G., Dickel, J. R. $\quad$ 1971, Astrophys. Letters, 8, 203.

189. Wilson, T. L., Altenhoff, W. 1970, Astrophys. Letters, $5,47$.

190. Wilson, T. L. 1970, IAU Symposium, 38, 140.

191. Wilson, W. J. 1971, Astrophys. J., 166, L13.

192. Yukimuk, A. K. 1971, Astrofizika (USSR), 7, 611.

193. Zabolotnyj, I. V. F. 1971 , Astron. Circ., 635, 1.

194. Zheleznyakov, V. V. 1971, Astrophys. Space Sci., 13, 74.

195. Backer, D. C. $\quad 1970$, Nature, 228, 42.

196. Backer, D. C. $\quad 1970$, Nature, 228, 752.

197. Backer, D. C. $\quad 1973$, Astrophys J., May issue.

\title{
C. LINE RADIATION FROM THE GALAXY
}

Ben Zuckerman

The goal of this section is to provide a complete bibliography of papers published in the past three years. A short list of various reviews and symposia is also included. The following discussion is an attempt to indicate what topic each reference is about. For a fuller discussion of the content of these papers, the reader is referred to the relevant reports to Commission 34 in the present volume and especially to the reviews listed below.

\section{21-Centimeter hydrogen line}

Hydrogen line absorption interferometry in the Northern (165) and Southern $(139,270,271,272$, 273) hemispheres has yielded important new information relating to the two-component models of $\mathrm{H}$ I regions. Surveys of various regions with single antennas have also been carried out in the Northern $(7,30,31,74,75,76,217,265,279,320,321,322,325,349,368,369,374,386,393)$ and Southern $(139,154,187,188,190,271,272,273)$ hemispheres. From these, various pictures of galactic structure $(32,33,34,95,167,197,227,228,289,290,291,292)$ are obtained. Specific sources have been examined $(18,19,45,57,92,109,110,123,124,125,138,162,166,170,184,185,193,202$, $232,277,302,306,347,348,371,377,379,380,391,416)$. The high velocity clouds $(62,87,168$, $192,195,205,206,231,242,276,372,381,384,387,388$ ) and high velocity gas near the galactic center $(203,204,234,307,308,309)$ have received special attention. 21-centimeter emission and/or absorption has been used to obtain pulsar distances $(86,126,127,143,144,225,304)$ and to study cold clouds and the abundance ratio of dust and gas $(221,268,279,293,303,305,324,339,373$, $385,392)$ and magnetic fields through the Zeeman effect $(24,370,375,376,382)$. Papers have been written on determination of the astronomical unit (244), 21-cm optical depths $(107,152)$, the total mass of atomic hydrogen gas in the galaxy (122), and lunar occultation in the $21-\mathrm{cm}$ line (201).

\section{Recombination lines}

The so-called 'diffuse' interstellar medium has recently been the object of considerable observational $(66,131,133,140,171)$ and theoretical $(40,130)$ work. Hydrogen lines from discrete $\mathrm{H}$ il regions in the Northern $(51,52,54,61,77,128,129,132,135,233,235,250,251,255,256,274,280,282,294$, 297, 298, 326, 337, 404, 405, 406) and Southern $(35,69,99,100,237,238,240,241,407)$ hemispheres have been investigated. Theoretical analysis of recombination lines from predominantly ionized $(4,5,20,21,22,23,83,117,156,157,158,159,160,253,263,318)$ and from predominantly 
neutral $(81,82,93,94,111,142,264,301)$ gas have been carried out. Planetary nebulae have been investigated with recombination lines $(116,161,295,341,342)$. Further helium abundances have been obtained $(16,53,239)$. Carbon $(80,239,254,423)$, hydrogen $(9,41,43,44,49)$ and other $(42$, 46, 47) recombination lines have been observed from $\mathrm{H}$ I regions. The galactic distribution of $\mathrm{H}$ iI has been investigated $(6,113,114,189)$.

\section{OH lines}

There have been general studies of the $\mathrm{OH}$ lines in radio continuum sources $(56,73,78,137$, $226,286,287,355,357,359,360)$, in optically dark clouds $(149,150,363,367,383)$, from infrared sources and stars $(36,37,48,96,252,281,283,285,409,410)$, and from the galactic center $(183$, 218, 219, 220, 288). Specific objects have been examined in considerable detail $(65,97,136,223$, $224,278,284,310,396,402,412,418)$. Interferometry $(58,64,84,145,146,147,148,273,410$, 411) has been carried out on the $\mathrm{OH}$ maser sources. An $\mathrm{OH}$ occultation was observed $(60)$ and the statistics of maser radiation studied (90). Searches for $\mathrm{OH}$ in various regions have been carried out $(191,198,413)$. Radiation from excited rotational levels has been observed $(101,103,104,249 \mathrm{a}$, $299,343,365,424,426)$ and searched for $(10,12,356) .{ }^{18} \mathrm{OH}$ has been studied $(14,98,408)$. Models for the maser radiation have been proposed $(2,17,91,118,119,120,172,209,210,211,212,213$, 214, 266). Laboratory measurements of the $\mathrm{OH}$ frequencies have been made $(10,55,236)$ as well as an astronomical estimate (222). An $\mathrm{OH}$ formation mechanism has been discussed (182).

\section{4. $\mathrm{H}_{2} \mathrm{CO}$ lines}

Formaldehyde has been studied at 6-cm wavelength in the direction of continuum sources (65, $108,207,315,395,401,403,420)$, optically dark nebulae $(207,327)$, the galactic center region $(105$, $106,183,310,314,316,317,394)$ and elsewhere (134). Models have been proposed to explain the anomalous cooling of the 6-cm transition $(210,248,344,350)$. An $\mathrm{H}_{2} \mathrm{CO}$ occultation was observed (194). The 2-mm lines from ortho and para- $\mathrm{H}_{2} \mathrm{CO}(208,346)$ and the $2-\mathrm{cm}$ line $(88)$ have been observed. The ${ }^{13} \mathrm{C}$ and ${ }^{18} \mathrm{O}$ isotopic forms have been studied $(102,394)$. Precise laboratory measurements of $\mathrm{H}_{2} \mathrm{CO}$ transition frequencies have been carried out $(\mathbf{2 4 6}, 352,353,354)$ as well as studies of $\mathrm{H}_{2} \mathrm{CO}$ photo dissociation rates $(\mathbf{1 1 2}, 338)$.

\section{Other molecules}

Observations have been made of $\mathrm{CO}(257,259,260,313,332,335,397,400)$, CN $(174,258,400)$, $\mathrm{H}_{2} \mathrm{O}(1,13,26,28,29,71,153,180,181,200,229,312,329,340,361,366)$, HCN (243, 330, 399, 415, 421), $\mathrm{SiO}(70,72,398), \mathrm{CH}_{3} \mathrm{OH}(11,15,362,399,425)$, $\mathrm{HNCO}(331), \mathrm{HC}_{3} \mathrm{~N}(358), \mathrm{NH}_{2} \mathrm{CHO}$ $(141,275,296), \mathrm{H}_{2} \mathrm{CS}(63,328), \mathrm{H}_{2} \mathrm{CO}_{2}(38,419), \mathrm{NH}_{3}(50,199,249,422)$, CS $(261,400,421)$, $\mathrm{CH}_{2} \mathrm{NH}$ (115), $\mathrm{H}_{2} \mathrm{~S}$ (345), $\mathrm{CH}_{3} \mathrm{CN}$ (333), $\mathrm{C}_{3} \mathrm{H}_{4}(331)$, OCS (176), and at least two unidentified lines $(25,27,196,331,421)$. A number of unsuccessful searches for other molecules have also been reported $(39,68,151,164,169,175,230,364)$, most notably searches for $\mathrm{H}_{2}{ }^{+}(85,173,323)$. Transition frequencies for $\mathrm{CH}(8), \mathrm{HC}_{3} \mathrm{~N}(67,177), \mathrm{HNCO}(163), \mathrm{H}_{2} \mathrm{CS}(178), \mathrm{H}_{2}{ }^{+}(215,336)$, $\mathrm{NO}(247), \mathrm{CH}_{3} \mathrm{OH}(269)$, and $\mathrm{HNO}(300)$ have been measured in the laboratory.

\section{Miscellaneous}

Searches have been carried out for a hyperfine structure line from ${ }^{3} \mathrm{He}(267,319)$ and a $3-\mathrm{cm}$ fine structure line of $H(245)$. Excitation of molecules has been investigated $(3,59,121)$ and molecule formation and destruction processes have been considered $(79,334,338,389,390,414)$. A search for intelligent life by means of monochromatic $927 \mathrm{MHz}$ emission was undertaken (351). 


\section{BIBLIOGR A P HY}

\section{Reviews and Symposia}

"Anomalous Emission from Interstellar Hydroxyl and Water," B. E. Turner, J. Royal Astr. Soc. Canada 64, 221 and 282, 1970.

"Radiofrequency Recombination Lines," A. K. Dupree and L. Goldberg, Ann. Rev. Astr. and Ap. 8, 231, 1970.

"Problems in Galactic Spiral Structure: An Account of a 'Spiral Workshop'." S. C. Simonson III, Astr. and $A p .9,163,1970$.

"Physical Conditions and Chemical Constitution of Dark Clouds," C. Heiles, Ann. Rev. Astr. and Ap. 9, 293, 1971.

“Interstellar Molecules and Dense Clouds.” D. M. Rank, C. H. Townes and W. J. Welch, Science 174, 1083, 1971.

"Microwave Receivers for Molecular Line Astronomy," D. Buhl and L. E. Snyder, Nature (Phys. Sci.) 232, 161, 1971.

“Microwave Celestial Water-Vapor Sources," K. J. Johnston, S. H. Knowles and P. R. Schwartz, Sky and Telescope, 44, 88, 1972.

Molecules and the Galactic Environment, Ed. M. A. Gordon and L. E. Snyder, Wiley and Sons, New York 1973 (in press).

Interstellar Dust and Related Topics, IAU Symposium 52, Ed. J. M. Greenberg and H. C. van de Hulst, Reidel, Dordrecht, 1973 (in press).

\section{Papers}

1. Akvilonova, A. B., et al. 1972 , Astr. Zhurnal, 49, 102.

2. Allen, L. and Peters, G. I. 1972, Nature (Phys. Sci), 235, 143.

3. Allison, A. C. and Dalgarno, A. 1971, Astron. and Ap., 13, 331.

4. Andrews, M. H. and Hjellming, R. M. 1969, Ap. Lett., 4, 159.

5. Andrews, M. H., et al. 1971, Ap. J., 167, 245.

6. Ariskin, V. I. 1971, Astr. Zhurnal, 48, 253.

7. Ariskin, V. I., et al. 1969, Astr. Zhurnal, 46, 1149.

8. Baird, K. M. and Bredohl, H. 1971, Ap. J., 169, L83.

9. Ball, J. A., et al. 1970, Ap. J., 162, L25.

10. Ball, J. A., et al. $\quad 1970, A . J ., 75,762$.

11. Ball, J. A., et al. 1970, Ap. J., 162, L203.

12. Ball, J. A., et al. $\quad 1971$, Ap. J., 163, L33.

13. Ball, J. A., et al. 1971, Ap. J., 163, 429.

14. Ball, J. A. and Penfield, H. 1970, Ap. J., 160, 349.

15. Barrett, A. H., et al. 1971, Ap. J., 168, L101.

16. Batchelor, A. S. J. and Brocklehurst, M. 1972, Ap. Lett., 11, 129.

17. Boyd, R. W. and Werner, M. W. 1972, Ap. J., 174, L137.

18. Bridle, A. H. and Kesteven, M. J. L. $\quad 1970$, A. J., 75, 902.

19. Bridle, A. H. and Kesteven, M. J. L. 1972, A. J., 77, 207.

20. Brocklehurst, M. $\quad 1970, M . N . R . A . S ., 148,417$. Also Nature, 225, 618.

21. Brocklehurst, M. and Leeman, S. $\quad 1971$, Ap. Lett., 9, 35.

22. Brocklehurst, M. and Seaton, M. J. 1971 , Ap. Lett., 9, 139.

23. Brocklehurst, M. and Seaton, M. J. $\quad$ 1972, M.N.R.A.S., 157, 179.

24. Brooks, J. W., et al. 1971 , Ap. Lett., 8, 121.

25. Buhl, D. and Snyder, L. E. 1970, Nature, 228, 267.

26. Buhl, D., et al. 1969, Ap. J., 158, L97.

27. Buhl, D. and Snyder, L. E. $1973, A p . J$. March issue.

28. Burke, B. F., et al. $\quad$ 1970, Ap. J., 160, L63.

29. Burke, B. F., et al. 1972, Astron. Zhurnal, 49, 465.

30. Burton, W. B. $\quad 1970$, Astron. and Ap. Suppl., $2,261$.

31. Burton, W. B. 1970, Astron. and Ap. Suppl., 2, 291.

32. Burton, W, B, 1971, Astron. and Ap., 10, 76.

33. Burton, W. B. 1972, Astron. and Ap., 16, 158.

34. Burton, W. B. 1972, Astron. and Ap., 19, 51. 
35. Caswell, J. L. $\quad$ 1972, Austral. J. Phys., 25, 443.

36. Caswell, J. L. and Robinson, B. J. 1970, Ap. Lett., 7, 75.

37. Caswell, J. L., et al. 1971, Ap. Lett., 9, 61.

38. Cato, M., et al. 1970, Ap. J., 160, L131.

39. Cato, T., et al. 1972, Astron. and Ap. 21, 435.

40. Cesarsky, C. J. and Cesarsky, D. A. 1971, Ap. J., 169, 293.

41. Cesarsky, D. A. 1971, Ap. J., 167, L89.

42. Chaisson, E. J. $1971, A p . J ., 167, \mathrm{~L} 61$.

43. Chaisson, E. J. $1971, A p . J ., 170,81$.

44. Chaisson, E. J. 1972, Nature (Phys. Sci.), 239, 83.

45. Chaisson, E. J. 1972, Astron. and Ap., 18, 149.

46. Chaisson, E. J. and Ball, J. A. 1971, Ap. J., 169, 495.

47. Chaisson, E. J., et al. 1972, Ap. J., 173, L131.

48. Chaisson, E. J. and Dickinson, D. F. 1972 , Ap. Lett., 12, 119.

49. Chaisson, E. J. and Goad, L. E. 1972, $A p$. J., 171, L61.

50. Cheung, A. C., et al. 1969, Ap. J., 157, L13.

51. Churchwell, E. 1971, Astron. and Ap., 15, 90.

52. Churchwell, E. and Edrich, J. 1970, Astron. and Ap., 6, 261.

53. Churchwell, E. and Mezger, P. G. 1970, Ap. Lett., 5, 227.

54. Churchwell, E., et al. 1970, Ap. Lett., 5, 157.

55. Churg, A. and Levy, D. H. 1970, Ap. J., 162, L161.

56. Coles, W. A. and Rumsey, V. H. 1970, Ap. J., 159, 247.

57. Colvin, R. S., et al. 1970, Ap. Lett., 6, 211.

58. Cooper, A. J., et al. 1971, M.N.R.A.S., 152, 383.

59. Crawford, O. H., et al. 1969 , Astron. and Ap., 2, 451.

60. Cudaback, D. D. and Welch, W. J. 1969, Ap. J., 155, L83.

61. Davies, R. D. 1971, Ap. J., 163, 479.

62. Davies, R. D. $\quad 1972$, Nature, $237,88$.

63. Davies, R. D., et al. 1971, M.N.R.A.S., 152, 7P.

64. Davies, R. D., et al. 1972, Nature (Phys. Sci.), 237, 21.

65. Davies, R. D. and Matthews, H. E. 1972, M.N.R.A.S., 156, 253.

66. Davies, R. D., et al. 1972, Nature (Phys. Sci.), 238, 101.

67. de Zafra, R. L. $1971, A p . J ., 170,165$.

68. de Zafra, R. L., et al. 1971, Ap. Lett., 10, 1.

69. Dickel, J. R. and Milne, D. K. 1972, Austral. J. Phys. 25, 539.

70. Dickinson, D. F. $1972, A p . J ., 175, \mathrm{~L} 43$.

71. Dickinson, D. F. and Chaisson, E. J. 1971, Ap. J. 169, 207.

72. Dickinson, D. F. and Gottlieb, C. A. 1971, Ap. Lett., 7, 205.

73. Dickinson, D. F. and Turner, B. E. 1972, Ap. Lett., 11, 1.

74. Dieter, N. H. 1971, Astron. and Ap., 59.

75. Dieter, N. H. 1972, Astron. and Ap. Suppl., 5, 21.

76. Dieter, N. H. 1972, Astron. and Ap. Suppl., 5, 313.

77. Doherty. L. H., et al. 1972, Ap. Lett., 12, 91.

78. Downes, D. 1970, Ap. Lett., 5, 53.

79. Duley, W. W. 1970, J. Roy. Astr. Soc. Canada, 64, 331.

80. Dupree, A. K. $\quad 1969$, Ap. J., 158, 491.

81. Dupree, A. K. $1971, A p . J ., 170, \mathrm{~L} 119$.

82. Dupree, A. K. $\quad 1972$, Ap. J., 173, 293.

83. Dyson, J. E. 1969, Ap. J., 155, 47.

84. Eliasson, B. and Bartlett, J. F. $\quad$ 1969, Ap. J., 155, L79.

85. Encrenaz, P. J. and Falgone, E. 1971, Ap. Lett., 8, 187.

86. Encrenaz, P. and Guèlin, M. 1970, Nature, 227, 476.

87. Encrenaz, P., et al. 1971, Astron. and Ap., 12, 16.

88. Evans, N. J. II, et al. $1970, A p . J ., 159, \mathrm{~L} 9$.

89. Evans, N. J. II, et al. 1970, Science, 169, 680.

90. Evans, N. J.II, et al. $\quad$ 1972, Phys. Rev. A, 6, 1643.

91. Evenson, K. M., et al. 1970, Phys. Rev. Lett., 25, 199.

92. Fejes, I. 1971, Astron. and Ap., 15, 419. 
93. Flannery, M. R. $\quad 1970$, Ap. Lett., 7, 85.

94. Flannery, M. R. $1970, A p . J ., 161, \mathrm{~L} 41$.

95. Fujimoto, M. and Tanahashi, Y. 1971, P.A.S. Japan, $23,7$.

96. Gahm, G. F. and Winnberg, A. 1971, Astron. and Ap., 13, 489.

97. Gardner, F. F. and McGee, R. X. 1971, Ap. Lett., 8, 83.

98. Gardner, F. F., et al. $\quad 1970$, Ap. Lett., 5, 67.

99. Gardner, F. F., et al. $\quad 1970$, Ap. Lett., 6, 87.

100. Gardner, F. F., et al. 1970 , Astron. and Ap., 7, 349.

101. Gardner, F. F. and Ribes, J. C. 1971, Ap. Lett., 9, 175.

102. Gardner, F. F., et al. 1971 , Ap. Lett., 9, 181.

103. Gardner, F. F., et al. $\quad 1970$, Ap. Lett., 7, 51.

104. Gardner, F. F., et al. 1971, Ap. J., 169, L109.

105. Gardner, F. F. and Whiteoak, J. B. 1970, Ap. Lett., 5, 161.

106. Gardner, F. F. and Whiteoak, J. B. 1972, Ap. Lett., 10, 171.

107. Gardner, F. F. and Whiteoak, J. B. $\quad$ 1972, Ap. Lett., 11, 123.

108. Gardner, F. F. and Whiteoak, J. B. 1972, Ap. Lett., 12, 107.

109. Garzoli, S. L. 1970, Astron. and Ap., 8, 7.

110. Garzoli, S. L. and Varsavsky, C. M. 1970, Ap. J., 160, 75.

111. Gayet, R., et al. 1969 , Astron. and Ap., 1, 365.

112. Gentieu, P. and Mentall, J. E. $\quad 1970$, Science, 169, 681.

113. Georgelin, Y. P. $\quad 1970$, Astron. and $A p ., 7,322$.

114. Georgelin, Y. P. and Georgelin, Y. M. 1971, Astron. and Ap., 12, 482.

115. Godfrey, P. D., et al. 1973 , Ap. Lett. (in press).

116. Goldberg, L. 1970, Ap. Lett., 5, 151.

117. Goldberg, L. and Cesarsky, D. 1970, Ap. Lett., 6, 93.

118. Goldriech, P. and Keeley, D. A. 1972, Ap. J., 174, 517.

119. Goldreich, P., et al. $\quad 1973, A p . J ., 179,111$.

120. Goldreich, P. and Kwan, J. Y. 1972, Ap. J., 176, 345.

121. Goldsmith, P. F. $\quad 1972, A p . J ., 176,597$.

122. Goldstein, S. J. Jr. $\quad 1972, A p . J ., 173,285$.

123. Goldstein, S. J. Jr. and MacDonald, D. D. $1969, A p . J ., 157,1101$.

124. Goniadski, D. $\quad 1972$, Astron. and Ap., 17, 378.

125. Gordon, C. P. $\quad 1970$, A. J., $75,914$.

126. Gordon, C. P., et al. 1969 , Nature, 222, 129.

127. Gordon, K. J. and Gordon, C. P. 1970, Ap. Lett., 5, 153.

128. Gordon, M. A. 1969, Ap. J., 158, 479.

129. Gordon, M. A. $\quad 1970$, Ap. Lett., 6, 27.

130. Gordon, M. A. $1971, A p . J ., 167,21$.

131. Gordon, M. A. and Cato, T. 1972, Ap. J., 176, 587.

132. Gordon, M. A. and Churchwell, E. 1970, Astron. and Ap., 9, 307.

133. Gordon, M. A. and Gottesman, S. T. 1971, Ap. J., 168, 361.

134. Gordon, M. A. and Roberts, M. S. 1971, Ap. J., 170, 277.

135. Gordon, M. A. and Wallace, D. C. 1971, Ap. J., 167, 235.

136. Goss, W. M., et al. 1971 , Astron. and Ap., 14, 481.

137. Goss, W. M., et al. 1970, Austral. J. Phys., 23, 559.

138. Goss, W. M. and Radhakrishnan, V. 1969, Ap. Lett., 4, 199.

139. Goss, W. M., et al. $\quad$ 1972, Ap. J. Suppl., 24, 123.

140. Gottesman, S. T. and Gordon, M. A. 1970, Ap. J., 162, L93.

141. Gottlieb, C. A., et al. 1973, Ap. J. June 15 issue.

142. Greenberg, D. W. 1969, Ap. J., 155, L51.

143. Guèlin, M., et al. 1933, Astron. and $A p ., 14,387$.

144. Guèlin, M., et al. 1969, Nature, 221, 249.

145. Habing, H. J., et al. 1972, Astron. and Ap., 17, 329.

146. Hardebeck, E. G. $\quad 1971, A p . J ., 170,281$.

147. Hardebeck, E. G. $\quad 1972, A p . J ., 172,583$.

148. Hardebeck, E. G. and Wilson, W. J. 1971, Ap. J., 169, L123.

149. Heiles, C. 1969, Ap. J., 157, 123.

150. Heiles, C. $\quad 1970$, Ap. J., $160,51$. 
151. Heiles, C. E. and Turner, B. E. $\quad 1971$, Ap. Lett., 8, 89.

152. Heiles, C. and Verschuur, G. L. 1969, Ap. Lett., 3, 21.

153. Hills, R., et al. 1972, Ap. J., 175, L59.

154. Hindman, J. V. and Kerr, F. J. 1970, Austral. J. Phys. Ap. Supp., 18, 43.

155. Hjellming, R. M., et al. $\quad 1969$, Ap. J., 157, 573.

156. Hjellming, R. M., et al. 1969, Ap. Lett., 3, 111.

157. Hjellming, R. M. and Churchwell, E. 1969, Ap. Lett., 4, 165.

158. Hjellming, R. M. and Davies, R. D. 1970, Astron. and Ap., 5, 1970.

159. Hjellming, R. M. and Gordon, M. A. 1971, Ap. J., 164, 47.

160. Huang-Binh, D. 1970, Ap. Lett., 6, 151.

161. Huang-Binh, D. 1971, Astron. and Ap., 10, 159.

162. Hobbs, L. M. 1971, Ap. J., 166, 333.

163. Hocking, W. H., et al. 1972, Ap. J., 174, L93.

164. Howard, W. E. III and Hvatum, H. 1969, Ap. J., 157, L161.

165. Hughes, M. P., et al. 1971, Ap. J. Supp., 23, 323.

166. Hughes, V. A. and Routledge, D. $\quad 1970, A . J ., 75,1148$.

167. Hughes, V. A. and Routledge, D. 1972, A. J., 77, 210.

168. Hulsbosch, A. N. M. 1971, Astron. and Ap., 14, 489 (erratum 15, 473).

169. Hvatum, H. and Howard, W. E. III 1970, $A p$. J., 162, L167.

170. Ilovaisky, S. A. 1971, Astron. and Ap., 11, 134.

171. Jackson, P. D. and Kerr, F. J. $\quad$ 1971, Ap. J., 168, 29.

172. Jefferies, J. T. 1971, Astron. and Ap., 12, 351.

173. Jefferts, K. B., et al. $\quad 1970, A p . J ., 159$, L15.

174. Jefferts, K. B., et al. $\quad 1970$, Ap. J., 161, L87.

175. Jefferts, K. B., et al. $\quad 1971$, Ap. Lett., 8, 43.

176. Jefferts, K. B., et al. 1971, Ap. J., 168, L111.

177. Johnson, D. R. and Lovas, F. 1971, Ap. J., 169, 617.

178. Johnson, D. R. and Powell, F. X. 1970, Science, 169, 679.

179. Johnston, K. J., et al. $\quad 1971$, Ap. J., 167, L93.

180. Johnston, K. J., et al. $\quad 1971$, Ap. J., 166, L21.

181. Johnston, K. J., et al. $\quad 1972$, Ap. Lett., 10, 93.

182. Julienne, P. S., et al. $1971, A p . J ., 170,65$.

183. Kaifu, N., et al. $\quad$ 1972, Nature (Phys. Sci)., 238, 105.

184. Kazes, I. 1971, Astron. and Ap., 15, 460.

185. Kazes, I. and Rieu, N. Q. 1970, Astron. and Ap., 4, 111.

186. Kerns, B. and Duncan, A. B. F. $\quad 1971$, Ap. J., 172, 331.

187. Kerr, F. J. $\quad$ 1969, Austral. J. Phys. Ap. Supp., 9, 1.

188. Kerr, F. J. and Hindman, J. V. $\quad 1970$, Austral. J. Phys. Ap. Supp., 18, 1.

189. Kerr, F. J. and Kerr, M. 1970, Ap. Lett., 6, 175.

190. Kerr, F. J. and Knapp, G. R. $\quad$ 1970, Austral. J. Phys. Ap. Supp., 18, 9.

191. Kerr, F. J. and Knapp, G. R. 1971, A. J., 76, 993.

192. Kerr, F. J. and Knapp, G. R. 1972, A. J., 77, 354.

193. Kerr, F. J. and Knapp, G. R. $1972, A . J ., 77,573$.

194. Kerr, F. J. and Sandquist, A. 1970, Ap. Lett., 5, 59.

195. Kerr, F. J. and Sullivan, W. T. III $1969, A p . J ., 158,115$.

196. Klemperer, W. 1970 , Nature, 227, 1230.

197. Knapp, G. R. 1972, Astron. and Ap., 21, 163.

198. Knapp, G. R. and Kerr, F. J. 1972, A. J., 77, 649.

199. Knowles, S. H. and Cheung, A. C. 1971, Ap. J., 164, L19.

200. Knowles, S. H., et al. 1969, Science, 166, 221.

201. Knowles, S. H. and Sullivan, W. T. III 1970, Ap. Lett., 6, 21.

202. Kovach, W. S. $1972, A p . J ., 173,287$.

203. Kruit, P. C. van der 1970, Astron. and Ap., 4, 462.

204. Kruit, P. C. van der 1971, Astron. and Ap., 13, 405.

205. Kuilenberg, J. van 1972, Astron and Ap. Suppl., 5, 1.

206. Kuilenberg, J. van 1972, Astron. and Ap., 16, 276.

207. Kutner, M. and Thaddeus, P. $\quad 1971, A p . J ., 168, \mathrm{~L} 67$.

208. Kutner, M., et al. $\quad 1971$, Ap. J., 164, L49. 
209. Litvak, M. M.

210. Litvak, M. M.

1969, Science, 165, 855.

1970, Ap. J., 160, L133.

1970, Phys. Rev. A, $2,937$.

211. Litvak, M. $M$.

1970, Phys. Rev. A, 2, 2107.

213. Litvak, M. M.

1971, Ap. J., 170, 71.

214. Litvak, M. M. and Dickinson, D. F. $\quad$ 1972, Ap. Lett., 12, 113.

215. Luke, S. K. $1969, A p . J ., 156,761$.

216. McCutcheon, W. H. 1970, Ap. Lett., 6, 221.

217. McCutcheon, W. H. and Shuter, W. L. H. 1970, A. J., 75, 910.

218. McGee, R. X. $\quad 1970$, Austral. J. Phys. Ap. Supp., 17, 1.

219. McGee, R. X. 1970 , Austral. J. Phys., 23, 541.

220. McGee, R. X., et al. $\quad$ 1970, Austral. J. Phys., 23777.

221. Mahoney, M. J. 1972, Ap. Lett., 12, 43.

222. Manchester, R. N. and Gordon, M. A. 1970, Ap. Lett., 6, 243.

223. Manchester, R. N. and Gordon, M. A. 1971, Ap. J., 169, 507.

224. Manchester, R. N., et al. 1969 , Ap. Lett., 3, 11.

225. Manchester, R. N., et al. $\quad$ 1969, Ap. Lett., 4, 229.

226. Manchester, R. N., et al. 1970 , Austral. J. Phys., 23, 751.

227. Mast, J. W. and Goldstein, S. J. Jr. $1970, A p . J ., 159,319$.

228. Mebold, U. 1972, Astron. and Ap., 19, 13.

229. Meeks, M. L., et al. 1969 , Science, 165, 180.

230. Meeks, M. L., et al. 1969, Science, 163, 173.

231. Meng, S. Y. and Kraus, J. D. $1970, A . J ., 75,535$.

232. Menon, T. K. $\quad 1970$, Astron. and Ap., 5, 240.

233. Menon, T. K. 1970, Ap. Lett., 7, 55.

234. Menon, T. K. and Ciotti, J. E. 1970 , Nature, 227, 579.

235. Menon, T. K. and Payne, J. 1969, Ap. Lett., 3, 25.

236. Meulen, ter J. J. and Dymanus, A. $\quad 1972, A p . J ., 172, \mathrm{~L} 21$.

237. Mezger, P. G., et al. 1970, Astron. and Ap., 4, 96.

238. Mezger, P. G., et al. 1970, Ap. Lett., 5, 117.

239. Mezger, P. G., et al. 1970, Ap. Lett., 6, 35.

240. Milne, D. K. and Wilson, T. L. 1971, Astron. and Ap., 10, 220.

241. Milne, D. K., et al. $\quad 1969$, Ap. Lett., 4, 121.

242. Minkowski, R., et al. 1972, Ap. J., 175, L123.

243. Morris, M., et al. $\quad 1971$, Ap. J., 170, L109.

244. Muhleman, D. O. $\quad 1969$, M.N.R.A.S., 144, 151.

245. Myers, P. C. and Barrett, A. H. 1972, Ap. J., 176, 111.

246. Nerf, R. B. Jr. $1972, A p . J ., 174,467$.

247. Neumann, R. M. $1970, A p . J ., 161,779$.

248. Oka, T. $1970, A p . J ., 160$, L69.

249. Oka, T., et al. 1971, Ap. J., 165, L15.

249a. Palmer, P. and Zuckerman, B. 1970, Ap. J., 161, L199.

250. Papadopoulos, G. D., et al. 1972 , Ap. Lett., 10, 89.

251. Parrish, A., et al. 1972, Ap. J., 178, 673.

252. Paschenko, M., et al. 1971 , Astron. and Ap., 11, 482.

253. Peach, G. $\quad 1972$, Ap. Lett., 10, 129.

254. Pedlar, A. 1970 , Nature, 226, 830.

255. Pedlar, A. and Davies, R. D. $\quad 1971$, Nature (Phys. Sci), 231, 49.

256. Pedlar, A. and Davies, R. D. 1972, M.N.R.A.S., 159, 129.

257. Penzias, A. A., et al. 1971, Ap. J., 165, 229.

258. Penzias, A. A., et al. $\quad 1972$, Phys. Rev. Lett., 28, 772.

259. Penzias, A. A., et al. $\quad 1972$, Ap. J. 178, L35.

260. Penzias, A. A., et al. $\quad 1972$, Ap. J., 174, L43.

261. Penzias, A. A., et al. $\quad 1971$, Ap. J., 168, L53.

262. Penzias, A. A., et al. 1970, A. J., 75, 141.

263. Percival, I. C. and Richards, D. 1969, Ap. Lett., 4, 235.

264. Percival, I. C. and Seaton, J. J. $\quad 1972$, Ap. Lett., 11, 31.

265. Perry, J. F. W. and Helfer, H. L. $\quad 1972, A p . J ., 174,341$. 
266. Peters, G. I. and Allen, L. 1972, Ap. J., 176, L23.

267. Predmore, C. R., et al. $\quad 1971$, Ap. J., 168, L125.

268. Quiroga, R. J. and Varsavsky, C. M. 1970, Ap. J., 160, 83.

269. Radford, H. E. 1972, Ap. J., 174, 207.

270. Radhakrishnan, V., et al. 1972, Ap. J. Supp., 24, 1.

271. Radhakrishnan, V. and Goss, W. M. 1972, Ap. J. Supp., 24, 161.

272. Radhakrishnan, V., et al. $\quad 1972$, Ap. J. Supp., 24, 49.

273. Radhakrishnan, V., et al. 1972, Ap. J. Supp., 24, 15.

274. Reifenstein, E. C. III, et al. $\quad$ 1970, Astron. and Ap., 4, 357.

275. Ribes, J. C., et al. 1972, Austral. J. Phys., (in press).

276. Rickard, J. J. 1971, Astron. and Ap., 11, 270.

277. Riegel, K. W. 1971, Ap. J., 164, 29.

278. Riegel, K. W. and Crutcher, R. M. 1972, Ap. J., 172, L107.

279. Riegel, K. W. and Crutcher, R. M. $\quad 1972$, Astron. and Ap., 18, 55.

280. Riegel, K. W. and Kilston, S. D. $\quad 1970, A p . J ., 159$, L155.

281. Rieu, N. Q., et al. 1971, Astron. and Ap., 14, 154.

282. Roberts, M. S. and Lockman, F. J. $1970, A p . J ., 161,877$.

283. Robinson, B. J., et al. $\quad 1971$, Ap. Lett., 8, 171.

284. Robinson, B. J., et al. 1970, Ap. Lett., 7, 79.

285. Robinson, B. J., et al. $\quad 1971$, Ap. Lett., 7, 163.

286. Robinson, B. J., et al. $\quad$ 1971, Ap. Lett., 9, 5.

287. Robinson, B. J., et al. $\quad$ 1970, Austral. J. Phys., 23, 363.

288. Robinson, B. J. and McGee, R. X. 1970, Austral. J. Phys., 23, 405.

289. Rohlfs, K. 1971, Astron. and Ap., 12, 43.

290. Rohlfs, K. $\quad 1971$, Astron. and Ap., 13, 46.

291. Rohlfs, K. 1972, Astron. and Ap., 16, 161.

292. Rohlfs, K. 1972, Astron. and Ap., 17, 246.

293. Rots, A. H., et al. 1972 , Astron. and Ap., 16, 344.

294. Rubin, R. H. and Mezger, P. G. 1970 , Astron. and Ap., 5, 407.

295. Rubin, R. H. and Palmer, P. 1971, Ap. Lett., 8, 79.

296. Rubin, R. H., et al. 1971, Ap. J., 169, L39.

297. Rubin, R. H. and Turner, B. E. $\quad 1969, A p . J ., 157, \mathrm{~L} 41$.

298. Rubin, R. H. and Turner, B. E. $\quad 1971, A p . J ., 165,471$.

299. Rydbeck, O., et al. $\quad 1970$, Ap. J., 161, L25.

300. Saito, S. and Takagi, K. 1972, Ap. J., 175, L47.

301. Salpeter, E. E. and Malone, R. C. 1971, Ap. J., 167, 27.

302. Sancisi, R. 1970, Astron. and Ap., 4, 387.

303. Sancisi, R. 1971, Astron. and Ap., 12, 323.

304. Sancisi, R. and Klomp, M. 1972, Astron. and Ap., 18, 329.

305. Sancisi, R. and Wesseling, P. R. $\quad 1970$, Astron, and Ap., 7, 34.

306. Sancisi, R. and Woerden, H. van 1970, Astron. and Ap., 5, 135.

307. Sanders, R. H. and Wrixon, G. T. 1972 , Astron and. Ap., 18, 92.

308. Sanders, R. H. and Wrixon, G. T. $\quad$ 1972, Astron. and Ap., 18, 467.

309. Sanders, R. H., et al. 1972 , Astron. and Ap., 16, 322.

310. Sandquist, A. S. 1970, A. J., 75, 135.

311. Sato, F. 1973, Publ. Astr. Soc. Japan, (in press).

312. Schwartz, P. R. and Barrett, A. H. $\quad 1970, A p . J ., 159, \mathrm{~L} 123$.

313. Schwartz, P. R. and Wilson, W. J. 1972, Ap. J., 177, L129.

314. Scoville, N. Z. $1972, A p . J .$, 175, L127.

315. Scoville, N. Z. and Solomon, P. M. 1973, Ap. J. 180, 31.

316. Scoville, N. Z. and Solomon, P. M. 1973, Ap. J. 180, 35.

317. Scoville, N. Z., et al. 1972 , Ap. J., 172, 335.

318. Sejnowski, T. J. and Hjellming, R. M. 1969, Ap. J., 156, 915.

319. Seling, T. V. and Heiles, C. 1969, Ap. J., 155, L163.

320. Shane, W. W. 1971, Astron. and Ap. Suppl., 4, 1.

321. Snahe, W. W. 1971, Astron. and Ap. Suppl., 4, 315.

322. Shane, W. W. 1972 , Astron. and Ap., 16, 118.

323. Shuter, W. L. H. and Sloan, D. S. $\quad$ 1969, Can. J. Phys., 47, 1233. 
324. Simonson, S. C. III $\quad$ 1970, Mem. Soc. Roy. Sci. Liège, 15th Ser., 19, 363.

325. Simonson, S. C. III, 1971, Astron. and Ap., 12, 136.

326. Simpson, J. P. 1970, Ap. Lett., 7, 43.

327. Sinclair, M. W. and Brooks, J. W. 1972, Ap. Lett., 11, 207.

328. Sinclair, M. W., et al. 1972 , Austral. J. Phys., (in press).

329. Snyder, L. E. and Buhl, D. 1969, Ap. J., 155, L65.

330. Snyder, L. E. and Buhl, D. $\quad 1971, A p . J ., 163$, L47.

331. Snyder, L. E. and Buhl, D. 1972, Annals. of the N Y. Academy of Sciences, 194, 17.

332. Solomon, P., et al, 1971, Ap. J., 163, L53.

333. Solomon, P. M., et al. $\quad 1971$, Ap. J., 168, L107.

334. Solomon, P. M. and Klemperer, W. 1972, Ap. J. 178, 389.

335. Solomon, P. M., et al. 1972, Ap. J. 178, 125.

336. Somerville, W. B. $\quad 1970$, M.N.R.A.S., 147, 201.

337. Sorochenko, R. L. and Berulis, J. J. 1969, Ap. Lett., 4, 173. Also Astr. Zhurnal 47, 850.

338. Stief, L. J., et al. 1972, Ap. J., 171, 21.

339. Sturch, C., 1969, A. J., 74, 82.

340. Sullivan, W. T. III 1971, Ap. J., 166, 321.

341. Terzian, Y. and Balick, B. $\quad 1969$, Ap. Lett., 4, 195.

342. Terzian, Y. and Balick, B. 1972, Ap. Lett., 10, 41.

343. Thacker, D. L., et al. $\quad 1970$, Ap. J., 161, L191.

344. Thaddeus, P. 1972, Ap. J., 173, 317.

345. Thaddeus, P., et al. $\quad$ 1972, Ap. J., 176, L73.

346. Thaddeus, P., et al. $\quad 1971$, Ap. J., 168, L59.

347. Thompson, A. R. and Colvin, R. S. $1970, A p . J ., 160,363$.

348. Thompson, A. R., et al. 1969, Ap. J., 158, 939.

349. Tolbert, C. R. $\quad 1971$, Astron. and Ap. Suppl., 3, 349.

350. Townes, C. H. and Cheung, A. C. 1969, Ap. J., 157, L103.

351. Troitskii, V. S., et al. $\quad 1971$, Astr. Zhurnal, 48, 645.

352. Tucker, K. D., et al. $\quad 1970$, Ap. J., 161, L153.

353. Tucker, K. D., et al. $\quad 1971$, Ap. J., 169, 429.

354. Tucker, K. D., et al. $\quad 1972$, Ap. J., 174, 463.

355. Turner, B. E. $\quad 1969, A$. J., 74, 985.

356. Turner, B. E. $\quad 1969$, Astron. and Ap., $2,453$.

357. Turner, B. E. $\quad 1970$, Ap. Lett., 6, 99.

358. Turner, B. E. $\quad 1971$, Ap. J., 163, L35.

359. Turner, B. E. $\quad 1971$, Ap. Lett., 8, 73.

360. Turner, B. E. $1972, A p . J ., 171,503$.

361. Turner, B. E. $\quad$ 1970, Astron. and Ap., 4, 165.

362. Turner, B. E., et al. 1972, Ap. J. 177, 609.

363. Turner, B. E. and Heiles, C. 1971, Ap. J., 170, 453.

364. Turner, B. E., et al. $\quad 1970$, Ap. Lett., 5, 197.

365. Turner, B. E., et al. 1970, Ap. J., 160, L125.

366. Turner, B. E. and Rubin, R. H. 1971, Ap. J., 170, L113.

367. Turner, B. E. and Verschuur, G. $\quad 1970, A p . J ., 162,341$.

368. Venugopal, V. R. and Shuter, W. L. H. 1969, M.N.R.A.S., 143, 27.

369. Venugopal, V. R. and Shuter, W. L. H. $\quad 1970$, Mem. R. Astr. Soc., 74, 1.

370. Verschuur, G. L. 1969 , Nature 223, 140.

371. Verschuur, G. L. $\quad 1969$, Astron. and Ap., 1, 473.

372. Verschuur, G. L. $\quad 1969$, Astron. and Ap., 3, 77.

373. Verschuur, G. L. $\quad 1969$, Ap. Lett., 4, 85.

374. Verschuur, G. L. $\quad 1969, A . J ., 74,597$.

375. Verschuur, G. L. $\quad 1969, A p . J ., 155, \mathrm{~L} 155$.

376. Verschuur, G. L. $\quad$ 1971, Ap. Lett., 7, 217.

377. Verschuur, G. L. $\quad 1970$, Ap. Lett., 6, 215.

378. Verschuur, G. L. $\quad 1970$, Ap. J., 161, 867.

379. Verschuur, G. L. $\quad 1970, A . J ., 75,687$.

380. Verschuur, G. L. $\quad 1971, A$. J., 76, 105.

381. Verschuur, G. L. $\quad 1971, A$. J., 76, 317. 


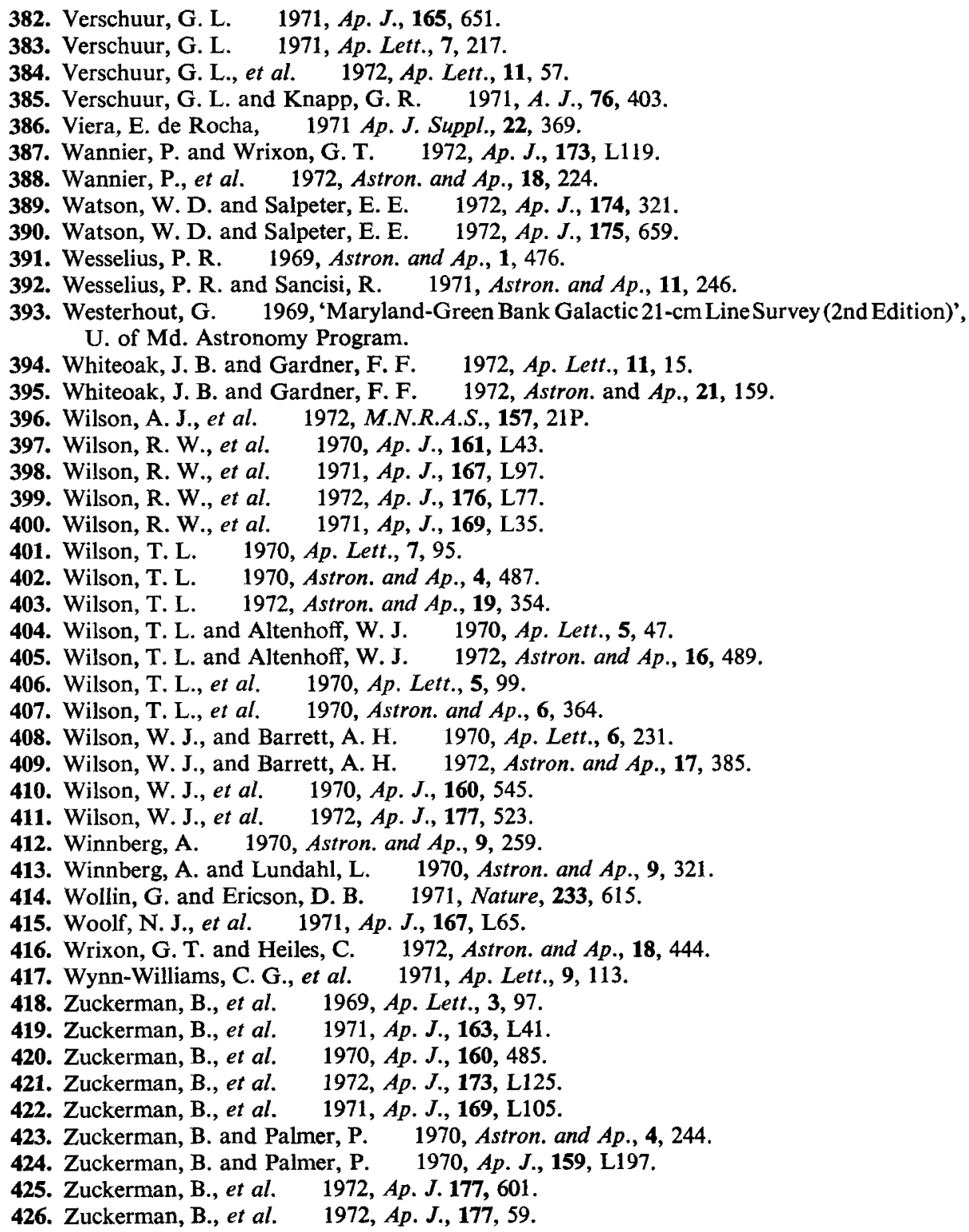

\section{EXTRAGALACTIC RADIO ASTRONOMY 1969-1972}

\section{H. van der Laan and G. K. Miley}

Radio astronomy shows increasing astrophysical diversity and the extragalactic branch ranges from spiral structure through relativistic astrophysics of nuclei of galaxies and quasars to observational cosmology. A bibliographic listing of about 700 papers under almost forty headings and subheadings has been prepared which systematically covers the subjects within our terms of reference. We list all relevant articles published in the journals listed in Section B(i), covering the period from July 1969 to November 1972. Comprehensive accounts of the subject may be found in the 